\title{
New Proline, Alanine, Serine Repeat Sequence for Pharmacokinetic Enhancement of Anti-VEGF Single-Domain Antibody
}

\author{
Farnaz Khodabakhsh, Morteza Salimian, Ardavan Mehdizadeh, Mohammad Sadeq Khosravy, \\ Alireza Vafabakhsh, Elmira Karami, and 을eza Ahangari Cohan \\ Department of Genetics and Advanced Medical Technology, Medical Biotechnology Research Center, Faculty of Medicine, AJA \\ University of Medical Sciences, Tehran, Iran (F.K.); Department of Medical Laboratory, Kashan University of Medical Sciences, \\ Kashan, Iran (M.S.); Department of Civil Engineering, Sharif University of Technology, Tehran, Iran (A.M.); Department of Rabies, \\ Virology Research Group (S.K.) and Department of Nanobiotechnology, New Technologies Research Group (R.A.C.), Pasteur \\ Institute of Iran, Tehran, Iran; and Department of Biology, School of Basic Science, Science and Research Branch, Islamic Azad \\ University, Tehran, Iran (A.V., E.K.)
}

Received March 23, 2020; accepted July 10, 2020

\begin{abstract}
Therapeutic fragmented antibodies show a poor pharmacokinetic profile that leads to frequent high-dose administration. In the current study, for the first time, a novel proline, alanine, serine (PAS) repeat sequence called PAS\#208 was designed to extend the plasma half-life of a nanosized anti-vascular endothelial growth factor-A single-domain antibody. Polyacrylamide gel electrophoresis, circular dichroism, dynamic light scattering, and electrophoretic light scattering were used to assess the physicochemical properties of the newly designed PAS sequence. The effect of PAS\#208 on the biologic activity of a single-domain antibody was studied using an in vitro proliferation assay. The pharmacokinetic parameters, including terminal half-life, the volume of distribution, elimination rate constant, and clearance, were determined in mice model and compared with the native protein and PAS\#1(200) sequence. The novel PAS repeat sequence showed comparable physicochemical, biologic, and pharmacokinetic features to the previously reported PAS\#1(200) sequence. The PAS\#208 increased the hydrodynamic radius and decreased significantly the electrophoretic mobility of the
\end{abstract}

native protein without any change in zeta potential. Surprisingly, the fusion of PAS\#208 to the single-domain antibody increased the binding potency. In addition, it did not alter the biologic activity and did not show any cytotoxicity on the normal cells. The PAS\#208 sequence improved the terminal half-life (14-fold) as well as other pharmacokinetic parameters significantly. The simplicity as well as superior effects on half-life extension make PAS\#208 sequence a novel sequence for in vivo pharmacokinetic enhancement of therapeutic fragmented antibodies.

\section{SIGNIFICANCE STATEMENT}

In the current study, a new proline, alanine, serine (PAS) sequence was developed that showed comparable physicochemical, biological, and pharmacokinetic features to the previously reported PAS\#1(200) sequence. The simplicity as well as superior effects on half-life extension make PAS\#208 sequence a novel sequence for in vivo pharmacokinetic enhancement of recombinant small proteins.

\section{Introduction}

Despite extensive researches in the field of cancer diagnosis and therapy, cancer remains one of the prominent causes of death in the world (Siegel et al., 2019). Angiogenesis is the most important and essential process in cancer development that leads to growth, metastasis, and invasion. It refers to the formation of new blood vessels from the existing vasculature. Angiogenesis inhibition interrupts the bloodstream to the

This project was financially supported by a grant from Iran National Science Foundation [Grant 97000450].

The authors declare there is no conflict of interest.

https://doi.org/10.1124/jpet.120.000012. tumor and causes oxygen and nutrient deficiencies in the tumor tissues (Lin et al., 2016). Vascular endothelial growth factor (VEGF) is a key regulator of angiogenesis process, which is expressed in the most solid tumors. Many investigations have been focused on blocking of the VEGF/ VEGFR2-signaling pathway that leads to the development of potent anticancer drugs. The most successful approved biopharmaceuticals for this purpose are anti-VEGF/VEGFR monoclonal antibodies (Zirlik and Duyster, 2018). Monoclonal antibodies and their fragments have now remarkably altered the outcome of cancer treatment in the clinic (Ecker et al., 2015). Although the conventional monoclonal antibodies have several clinical advantages, they suffer from immune system

ABBREVIATIONS: AUC, area under the curve; CL, clearance; HEK293, human embryonic kidney 293; HRP, horseradish peroxidase; MDA-MB231, M.D. Anderson-metastasis breast-cancer; Nb, nanobody; PAS, proline, alanine, serine; PEG, polyethylene glycol; rhVEGFA ${ }_{165}$, recombinant human VEGFA 165; RT, room temperature; $T_{1 / 2}$, terminal half-life; VEGF, vascular endothelial growth factor; VEGFR, VEGF receptor; $V_{d}$, volume of distribution. 
stimulation and low accessibility to the hidden antigens in the solid tumors (Muyldermans, 2013; Mendler et al., 2016). Recently, there is great attention to next-generation antibodies. Single-domain antibodies, also known as nanobodies, are antibody fragments derived from Camelidae heavy-chain antibodies. Single-domain antibodies are the smallest antigen-binding fragments that provide many advantages in comparison with the commercially available antibodies, such as small size, low immunogenicity, high affinity, high solubility, high stability, high expression in the prokaryotic systems, and easy manipulation (Iezzi et al., 2018). Moreover, they can easily penetrate to the solid tumors because of their small sizes (Van Audenhove and Gettemans, 2016). However, similar to other small-sized therapeutic proteins, they require high-dose administration and/or repeated injections to reach the therapeutic window in the circulation. This is caused by a rapid clearance via glomerular filtration that affects the treatment efficacy, patient compliance, and bystander effects (Ahmadpour and Hosseinimehr, 2018). Several strategies have been developed to extend the plasma half-life of small therapeutic proteins (Dingermann, 2013; Brandl et al., 2019). Polyethylene glycol (PEG)-ylation is a successful strategy because several PEGylated biopharmaceuticals are currently approved and available in the market (Brandl et al., 2019). The noticeable features of PEG, high hydrophilicity, and random coil conformation in the aquatic environments increase the hydrodynamic radius of protein above the renal filtration threshold $(6-8 \mathrm{~nm}$ ) (Longmire et al., 2008; Cohan et al., 2011). However, despite there being many available PEGylated drugs in the market, PEGylation technology has several disadvantages, including accumulation of PEG polymer in the kidney, high cost of production, high polydispersity, nonbiodegradability, tendency to raise neutralizing anti-PEG antibodies, high viscosity in PEG formulations, and difficult manufacturing processing (Gebauer and Skerra, 2018). Recently, PEG mimetic peptides have been developed based on recombinant DNA technology. It extends the half-life of small-sized biopharmaceuticals in the circulation similar to PEG without problems associated with PEG polymer. Although initially designed PEG mimetic peptides had deleterious effects on the biologic activity and solubility of fused protein (Kontermann, 2012; Schmidt, 2013), recent advances led to design of peptide sequences without the mentioned problems. In the last developed technology, proline, alanine, serine (PAS)-ylation, a hydrophilic random coil sequence of proline, alanine, and serine repeats (100-600 amino acids) was genetically fused either to the $\mathrm{N}$ terminus or $\mathrm{C}$ terminus of desired protein (Ahmadpour and Hosseinimehr, 2018). In contrast with albumin and Fc fusion technologies, desired pharmacokinetic parameters can be achieved in a controlled manner (Kontermann, 2012). Moreover, the PAS sequence provides monodisperse fusion proteins with a definite size and unique pharmacokinetic profile. Designed PAS sequences are nonimmunogenic, fully biodegradable, highly soluble, without accumulation in normal organs, resistant to the plasma proteases, easily produced in prokaryotic systems, and of a random coil structure without negative or positive charges (Schellenberger et al., 2009; Schlapschy et al., 2013; Podust et al., 2016; Gebauer and Skerra, 2018).

In the current study, for the first time, a novel PAS sequence, called PAS\#208, was employed to extend the plasma half-life of a nanosized anti-VEGFA single-domain antibody. The physicochemical properties, in vitro biologic activity, as well as pharmacokinetic parameters of the developed PASylated nanobody were compared with the native protein.

\section{Materials and Methods}

Chemical Reagents and Media. Anti-VEGFA nanobody in pHEN6 expression plasmid was gifted from Department of Biotechnology, Pasteur Institute of Iran. Recombinant human VEGFA165 (rhVEGFA $\left.{ }_{165}\right)$ and HRP-conjugated anti-His antibody were purchased from R\&D Systems (Minneapolis, MN) and Roche (Basel, Switzerland), respectively All other chemicals were obtained from Merck (Darmstadt, Germany). MDA-MB-231 and human embryonic kidney 293 (HEK293) cells were purchased from the national cell bank (Pasteur Institute of Iran) and cultured in Dulbecco's modified Eagle's medium supplemented with $2 \% \mathrm{FBS}$ in a $37^{\circ} \mathrm{C}$ incubator with $5 \% \mathrm{CO}_{2}$. Female BALB/c mice (20 g in weight) were also obtained from Pasteur Institute of Iran, and animal study was in accordance with the Helsinki Declaration.

Design of PAS\#208 Sequence. We checked several previously reported PAS sequences, including PAS\#1, PAS\#2, PAS\#3, PAS\#4, PAS\#5, and PAS\#1P2, which are different in the percentage of proline, alanine, and serine amino acids as well as the order of residues. After structural investigation on how these amino acids could increase the volume of the molecule, we finally purposed APSPASPA sequence for enhancing the pharmacokinetic parameters of an anti-VEGF nanobody. A computational method was used to check the volume of PAS\#208 before the experimental step, which is essential for pharmacokinetic enhancement of small-sized therapeutic proteins (Kontermann, 2011). Briefly, the 3D-model of PAS\#208 polypeptide was built by I-TASSER server (Roy et al., 2010). The generated model was then subjected to all-atom molecular dynamics simulation using allatom optimized potentials for liquid simulations force-field GROMACS package version 2016.3 in a Linux Mint 17 operating system (Hess et al., 2008). The simulation was continued until the root-meansquare deviation of the protein was reached at a plateau. The 3D model was placed at the center of a cubic box with $1.0 \mathrm{~nm}$ from the boundary, and the periodic boundary condition was applied. An extended simple point charge (SPCE) model was used as a water model, and the system was neutralized by adding either $\mathrm{Na}^{+}$or $\mathrm{Cl}^{-}$ ions. The temperature and pressure were kept at $300 \mathrm{~K}$ and one bar using Berendsen thermostat and Parrinello-Rahman barostat algorithms, respectively. Finally, the volume of PAS\#208 polypeptide was measured using gmx sasa command.

Expression and Purification of the Fusion Protein. PAS\#208 sequence was C-terminally fused to the anti-VEGFA nanobody. Moreover, a His-tag sequence was added to the $\mathrm{C}$ terminus of fusion protein for identification and purification. The construct was ordered to synthesize and clone into pET-26b(+) vector by Biomatik (Cambridge, Canada) using NdeI and EcoRI restriction enzymes. The expression plasmid was then transformed to BL21 (DE3) Escherichia coli strain using heat shock protocol. For protein expression, a positive clone was cultured in Terrific broth supplemented with $50 \mathrm{mg} / \mathrm{ml}$ kanamycin. The expression was induced by adding $1 \mathrm{mM}$ isopropyl $\beta$-d-1-thiogalactopyranoside $\left(\mathrm{OD}_{600 \mathrm{~nm}}=0.5\right)$, and the culture was incubated for 16 hours at $30^{\circ} \mathrm{C}$. The bacterial cells were then centrifuged at $12,000 \mathrm{~g}$ for 5 minutes and disrupted by adding 2-\{[2-hydroxy-1,1-bis(hydroxymethyl)ethyl]amino\}ethanesulfonic acid buffer $(0.5 \mathrm{M}$ sucrose, $1 \mathrm{mM}$ EDTA, and $0.1 \mathrm{M}$ Tris/HCl, adjusted to $\mathrm{pH}$ 8.0). The fusion protein was purified using $\mathrm{Ni}$-nitrilotriacetic acid affinity chromatography according to the instruction (Qiagen, Germany). The buffer was exchanged, and the protein was concentrated by ultrafiltration (3000 kDa cut-off; Millipore). Similar steps were done for the expression and purification of anti-VEGF nanobody. The expression of both proteins was studied by $15 \%$ SDS-PAGE and Coomassie Brilliant Blue staining method. For protein identification, 
the lysates were blotted into nitrocellulose membranes (Bio-Rad, Hercules, CA) and then blocked by skim milk $2.5 \%$ in Tris-buffered saline/Tween 20 (0.02 M Tris, $0.5 \mathrm{M} \mathrm{NaCl}, 0.05 \%$ Tween20, adjusted to $\mathrm{pH}$ 7.4) overnight at $4^{\circ} \mathrm{C}$. Thereafter, the blocked membranes were washed three times using Tris-buffered saline/Tween 20 buffer and incubated with anti-His HRP-conjugated antibody (1: 2000 dilution; Roche) for 1 hour at $37^{\circ} \mathrm{C}$. Finally, the membranes were stained with 3,3'-diaminobenzidine (Sigma) according to the instructions. The protein concentrations in all steps were measured using Bradford assay (Bradford, 1976).

Electrophoretic Mobility Assay. PAGE (15\%) and Coomassie Brilliant Blue staining method were used for electrophoretic mobility assessment of PASylated nanobody in comparison with the native protein. The electrophoretic mobility of purified proteins was defined as the distance traveled by the proteins through the polyacrylamide gel (Kim et al., 2006).

Circular Dichroism Spectroscopy. The change in the secondary structure of nanobody after fusion to the PAS\#208 sequence was analyzed using a spectropolarimeter (JASCO Corporation, Tokyo, Japan). The spectra were recorded at 190-250 nm using $0.5 \mathrm{mg} / \mathrm{ml}$ protein solutions at room temperature (bandwidth $1 \mathrm{~nm}$, scan speed $200 \mathrm{~nm} / \mathrm{min}$, response 1 second). Finally, the molar ellipticity (QM) was calculated for both proteins using the following equation:

$$
\mathrm{QM}=1 / 4 \text { Qobs } /(\mathrm{cd})
$$

in which Qobs is the measured ellipticity, $\mathrm{c}$ is the protein concentration $(\mathrm{M})$, and $\mathrm{d}$ is the path length of the cuvette $(\mathrm{cm})$ (Zvonova et al., 2017).

Size Distribution and Zeta Potential. Size distributions and zeta potential of nanobody after fusion to the PAS\#208 sequence were measured using Zetasizer Nano ZS (Malvern) at a concentration of $0.3 \mathrm{mg} / \mathrm{ml}$.

Binding Potency. The binding potency to rhVEGFA $_{165}$ was determined by a homemade ELISA. Briefly, $1 \mu \mathrm{g}$ rhVEGFA 165 was coated in each well, and the wells were blocked with skim milk solution (3\% w/v in PBS) for 1 hour at RT. After being washed three times with PBS/Tween, different concentrations of nanobody and the fused form were added, and the plates were further incubated for 1 hour at RT. The plates were then washed with PBS/Tween and incubated with anti-His HRP-conjugated antibody (1:2000 dilution; Roche) for 1 hour at RT. Finally, the substrate (3,3',5,5' -tetramethylbenzidine; PishtazTeb, Iran) was added, and the absorbance was measured at a wavelength of $450 \mathrm{~nm}$ using a spectrophotometer (BioTeK). Four-parameter logistic regression method was used for curve fitting. The $\mathrm{EC}_{50}$ values were calculated by Prism 8 software (GraphPad Software) after curve fitting.

VEGFA Neutralization. VEGFA neutralization was assessed on MDA-MB-231 cells in vitro. HEK293 cells were also used as a VEGFR2 negative cell line. Different concentrations of nanobody and the PAS\#208 fused form were incubated with $50 \mathrm{ng} / \mathrm{ml} \mathrm{rhVEGFA}{ }_{165}$ for 4 hours at $37^{\circ} \mathrm{C}$. The mixtures were then added to the cultured cells and incubated for 72 hours. After incubation, 3-(4,5-dimethylthiazol-2-yl)-2,5-diphenyl tetrazolium bromide solution $(5 \mathrm{mg} / \mathrm{ml})$ was added to each well and incubated for 4 hours at $37^{\circ} \mathrm{C}$. The crystalline dye was finally solubilized by adding DMSO, and the absorbance was measured at a wavelength of $570 \mathrm{~nm}$ using a spectrophotometer (BioTeK). Two-way ANOVA was used for statistical analysis of VEGF-neutralizing assay.

Pharmacokinetic Study. BALB/c mice were randomly divided into two groups (nanobody and PASylated nanobody, $n=24$ ) and received an intravenous dose of $5 \mathrm{mg}$ protein $/ \mathrm{kg}$. The blood samples were collected after 5,15 , and 30 minutes and $1,2,3,4,5,6,8,24,48$, and 72 hours of injection. The plasma was taken by centrifugation at $12,000 \mathrm{~g}$ for 25 minutes at $4^{\circ} \mathrm{C}$. The protein concentrations were measured by a homemade ELISA as previously described. The plasma from PBS-receiving mice was used as blank. A two-phase decay regression method was used for the curve fitting. The terminal half-life $\left(\mathrm{T}_{1 / 2}\right)$ was determined using linear regression of the last three plasma concentrations. The mean area under the curve $\left(\mathrm{AUC}_{0-\infty}\right)$ was determined using a linear trapezoidal method with extrapolation to infinity. Clearance $(C L)$ and volume of distribution $\left(\mathrm{V}_{\mathrm{d}}\right)$ values were also calculated (Cohan et al., 2011; Maleki et al., 2012).

Statistical Analysis. Curve plotting and data analysis were carried out using Prism 8 (GraphPad Software). A $P$ value of less than 0.05 was considered a statistically significant difference between the groups.

\section{Results}

Design of PAS\#208 Sequence. The 3D model of PAS\#208 polypeptide was generated, and the molecular dynamics were investigated in an aqueous solution for $100 \mathrm{~ns}$ (Fig. 1). The proposed PAS\#208 sequence had a volume of $18,205 \AA^{3}$, which was comparable to that of previously reported PAS sequences (Skerra et al., 2013).

Electrophoretic Mobility. Periplasmic PAS\#208-Nb and nanobody were purified using nickel affinity chromatography. The electrophoretic assessment on $15 \%$ gel showed lower mobility of PASylated nanobody at $\sim 75 \mathrm{kDa}$ (Fig. 2A). A band around $15 \mathrm{kDa}$ was observed in the gel for nanobody. The identification of PAS\#208-Nb was performed using an antiHis monoclonal antibody (Fig. 2B).

Size Distribution and Zeta Potential. The hydrodynamic radius and zeta potential of PAS\#208-Nb were studied using dynamic light scattering and electrophoretic light scattering, respectively. The hydrodynamic radius of nanobody increased 5.3-fold by the attachment of PAS\#208 sequence (Fig. 3). Size distribution analysis showed peaks at $77 \mathrm{~nm}$ (Khodabakhsh et al., 2018) and $409 \mathrm{~nm}$ for the native and the PASylated form, respectively. In contrast, no significant difference was observed in zeta potential after the PAS\#208 fusion. The observed values were -2.8 and $-2.4 \mathrm{mV}$ for PAS\#208-Nb and nanobody, respectively.

Secondary Structure. The secondary structure of the proteins was studied using circular dichroism spectroscopy. Molar ellipticity was calculated and plotted against wavelengths. A negative shift was observed for PAS\#208-Nb in the spectrum at a wavelength of $204 \mathrm{~nm}$ (Fig. 4), indicating an increase in random coil conformation of the protein.

Binding Potency. The binding potency of nanobody after fusion to PAS\#208 was determined by a homemade ELISA. After curve fitting, the $\mathrm{EC}_{50}$ values were calculated as $2.01 \times$ $10^{-7}$ and $1.76 \times 10^{-5} \mathrm{M}$ for PAS\#208-Nb and nanobody, respectively. The data revealed that the PAS\#208 sequence positively affects the antigen-binding potency of nanobody with a saturation state at lower concentrations (Fig. 5).

VEGFA Neutralization. VEGFA neutralization of PAS\#208-Nb and nanobody were investigated on MDA-MB231 breast-cancer cells. HEK293 cells were used as a control because of a lack of VEGFR2 expression. PAS\#208-Nb and nanobody showed the same VEGFA-neutralizing effect on MDA-MB-231 breast-cancer cells (Fig. 6A). These data indicated that PAS\#208 did not have a deleterious effect on the neutralizing efficacy of nanobody. In addition, all proteins did not show any effect on HEK293 because of lacking VEGFR2 even after 72 hours treatment (Fig. 6B).

Pharmacokinetics. The plasma concentration of the proteins was determined at defined time intervals, and a twophase decay model was used for curve fitting of data (Fig. 7). 


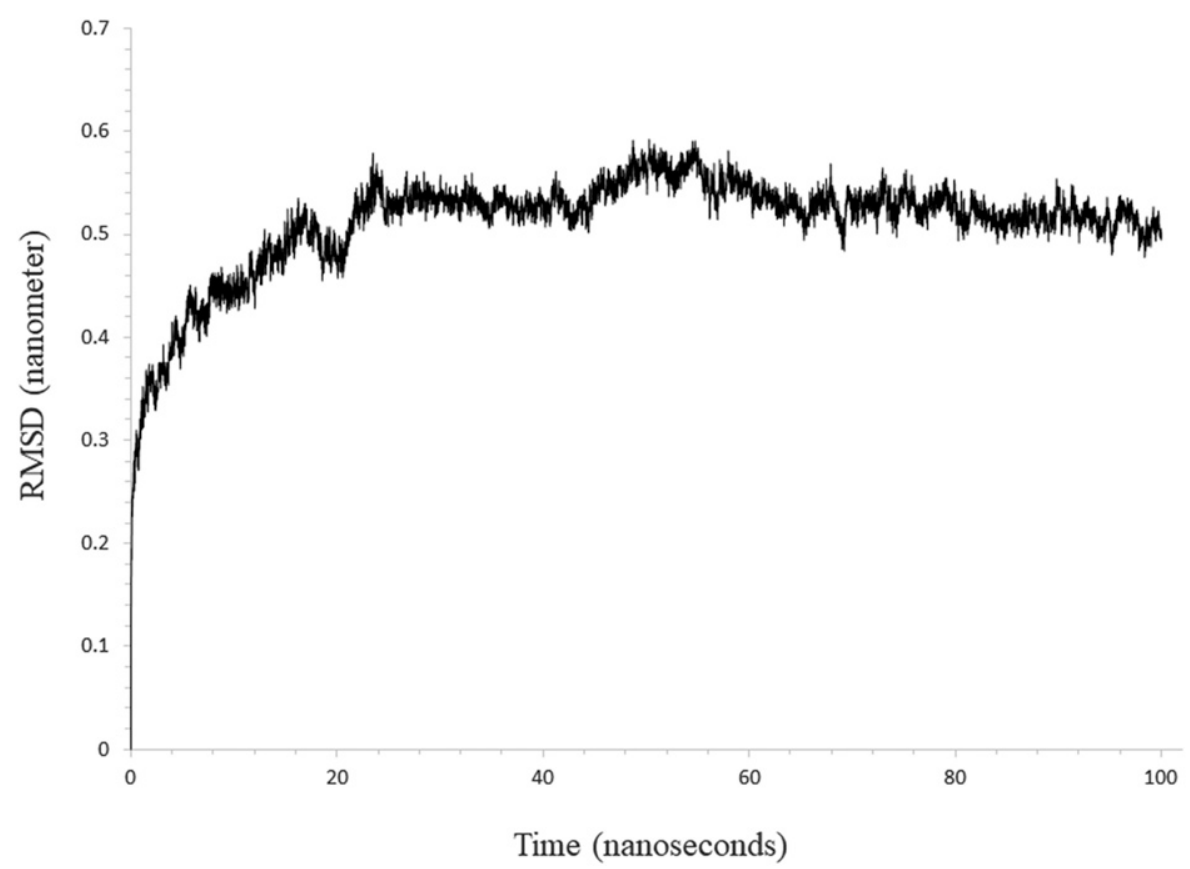

Fig. 1. Root-mean-square deviations (RMSDs) of PAS\#208 polypeptide during 100 nanoseconds molecular dynamics simulation.

Pharmacokinetic parameters, including $\mathrm{T}_{1 / 2}$, the $\mathrm{V}_{\mathrm{d}}$, elimination rate constant $(\beta)$, and $\mathrm{CL}$, were calculated. The terminal half-life of PAS\#208-Nb was increased 14-fold in comparison with the native protein. Moreover, $\mathrm{V}_{\mathrm{d}}$, CL, $\beta$, and AUC parameters were enhanced for the PASylated nanobody by 0.01-, 0.06-, 0.07-, and 24.1-fold, respectively (Table 1).

\section{Discussion}

Despite several advantages of antibody fragments, very short in vivo half-life is a critical point to apply them popularly in the clinic. To overcome this obstacle, different strategies have been developed. Recently, a new promising strategy called PASylation genetically links repeats of unstructured sequences of proline, alanine, serine to the gene of interest and remarkably enhances the pharmacokinetic parameters in vivo by a reduction in glomerular filtration rate (Skerra et al., 2013). Moreover, it can be easily established in the prokaryotic expression systems because it has no need for post-translational modifications (Schlapschy et al., 2013).

In the current study, for the first time, we designed a new PAS sequence called PAS\#208. Then, physicochemic and in vitro biologic characteristics and in vivo pharmacokinetic parameters were tested by the fusion to an anti-VEGF singledomain antibody. Similar to PAS\#1(200) sequence, the deigned PAS\#208 noticeably improved the physicochemic properties of the fused fragmented antibody. Because of the hydrophilic nature of the PAS\#208 sequence, like other same length PAS
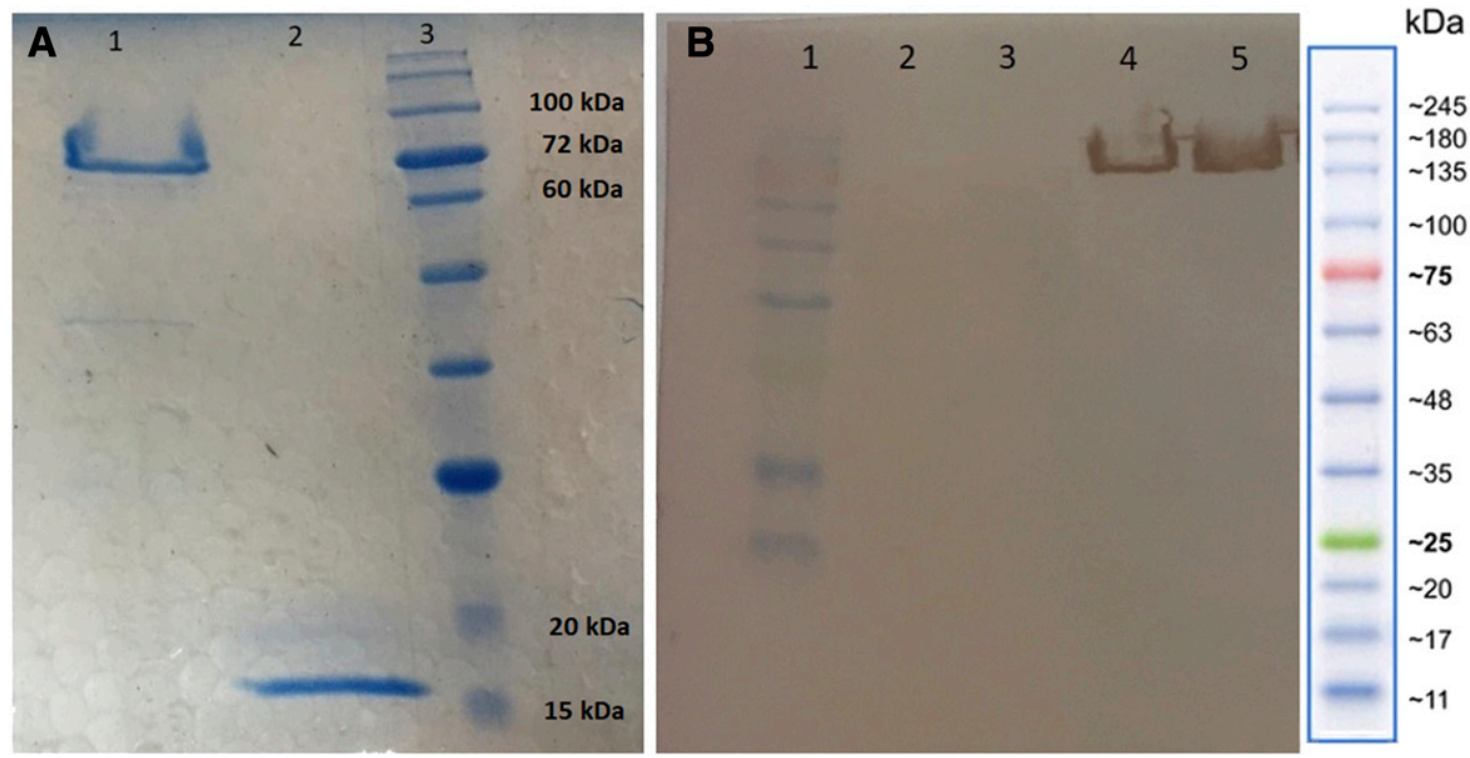

Fig. 2. (A) Electrophoretic mobility of purified PAS\#208-Nb and nanobody on 15\% gel (lane 1: nanobody; lane 2: PAS\#208-Nb; lane 3: marker). (B) Western blot analysis of PAS\#208-Nb (lane 1: marker; lanes 2 to 3: PAS\#208-Nb clones before induction; lanes 4 to 5: PAS\#208-Nb clones after induction with $1 \mathrm{mM}$ isopropyl $\beta$-d-1-thiogalactopyranoside). 


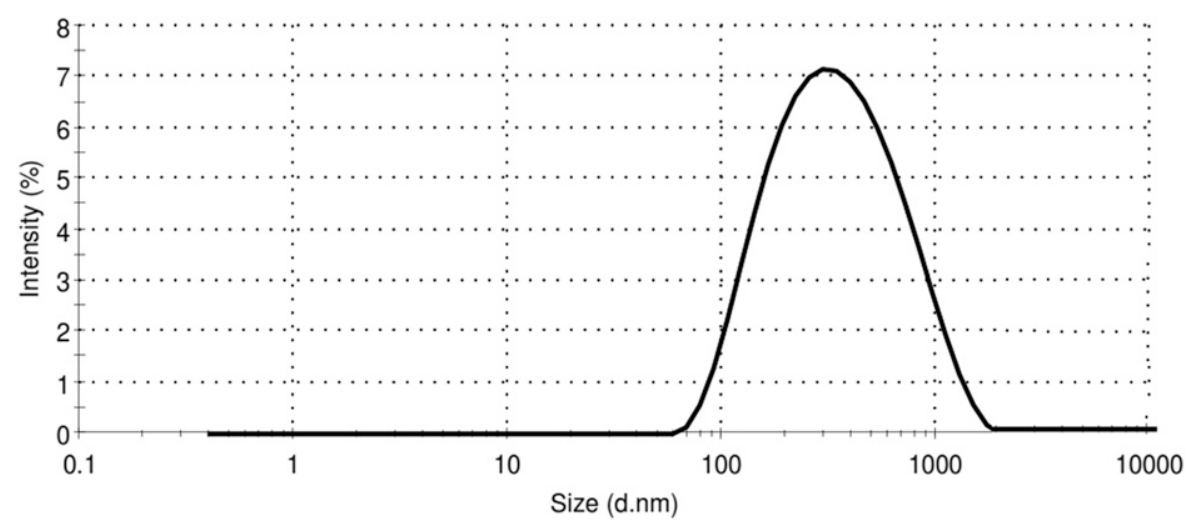

Fig. 3. Size distribution analysis of PAS\#208$\mathrm{Nb}$ using dynamic light scattering technique. sequences, the electrophoretic mobility of fusion protein showed a 2 -fold decrease in the electric field. This phenomenon can be explained by the lower binding affinity between the hydrophobic SDS and hydrophilic PAS\#208 sequence in the polyacrylamide gel electrophoresis. As shown, the negative charge of SDS is the main driving force for the mobility of proteins in the gel networks (Schlapschy et al., 2013; Zvonova et al., 2017). Moreover, lower electrophoretic mobility can also be related to the unstructured nature of the PAS\#208 sequence. As revealed by circular dichroism, an increase in random coil structure was observed similar to the other investigations on PAS\#1 sequence. This highly flexible random conformation subsequently increases the occupied volume of the fusion protein in water, which makes it harder to pass through the pores of the gel (Schlapschy et al., 2013; Morath et al., 2015; Zvonova et al., 2017).

Size distribution measurement revealed a great increase in the hydrodynamic radius of the protein after the fusion to PAS\#208. However, in contrast to Fc or albumin fusion technologies, it did not limit the penetration rate of the drugs into the targeted tissues because of the high flexibility as well as the controllable size of PAS sequences. In addition, a lower aggregation rate was observed in comparison with the native protein. The exposure of polar groups like hydroxyl groups of serine residues as well as other atoms that can be involved in donor or acceptor hydrogen binding in the PAS\#208 sequence can explain the lower aggregation rate (Schlapschy et al., 2013). Zeta potential measurement indicated PAS\#208 fusion did not change the net charge of the native protein, which was similar to our previous data obtained by the fusion of PAS\#1(200) to the anti-VEGF single-domain antibody (Khodabakhsh et al., 2018). Similar observations were also reported by other studies that PAS sequences did not alter the charge because no ionizing groups exist in the designed PAS sequences (Schlapschy et al., 2013). This is very important in the manufacturing of PASylated proteins because no variation is needed for downstream processes, which prevent additional costs in the production. Additionally, it was shown that the coupling of negative or positive moieties to the drugs severely affects the drug distribution as well as affinity to the receptor like glutamic acid-rich sequences (XTEN moiety) (Schlapschy et al., 2013).

Similar to other investigations on different PAS sequences, molecular simulations revealed the PAS\#208 sequence shows a random coil conformation without deleterious effects on the secondary structure of nanobody, which was experimentally confirmed by circular dichroism. This random coil structure did not affect the receptor-binding site of the native protein after the $\mathrm{C}$ terminus fusion to PAS\#208 as shown by the binding potency test. Because the PAS\#208 sequence acts as a flexible linker, no linker is necessary to fuse this sequence to the desired proteins to keep the affinity. Other half-life extension technologies often reduce the binding affinity of the native protein.

Although major changes were made in the physicochemical characteristics of PAS\#208-Nb, in vitro antiproliferative activity of the native remained intact after the fusion to PAS\#208. Nanobody and PAS\#208-Nb could equally inhibit the propagation of breast-cancer cells (MDA-MB-231) expressing VEGFR2 on the cell surface. These results are similar to those from our study on this protein that fused to PAS\#1(200) sequence. Similarly, nanobody and PAS\#208-Nb did not show any cytotoxicity on HEK293 cells because no VEGFR2 expression was found on the cell surface (Khodabakhsh et al., 2018). Different technologies were developed to compensate for the short in vivo half-life of miniaturized antibodies for medicinal purposes, such as antialbumin nanobody conjugation, PEGylation, and biodimerization (Vugmeyster et al., 2012; Hoefman et al., 2015; Van Roy et al., 2015). However, these approaches usually have unpredictable/deleterious effects on the physicochemical or biologic properties of the desired protein. In addition, in some cases, these approaches are usually hard to establish at the industrial scale and include difficult manufacturing processes. Long-acting biopharmaceuticals can easily produce by PASylation technology that improves the pharmacokinetic parameters of protein in a controlled manner by adding repeated sequences at the genetic level

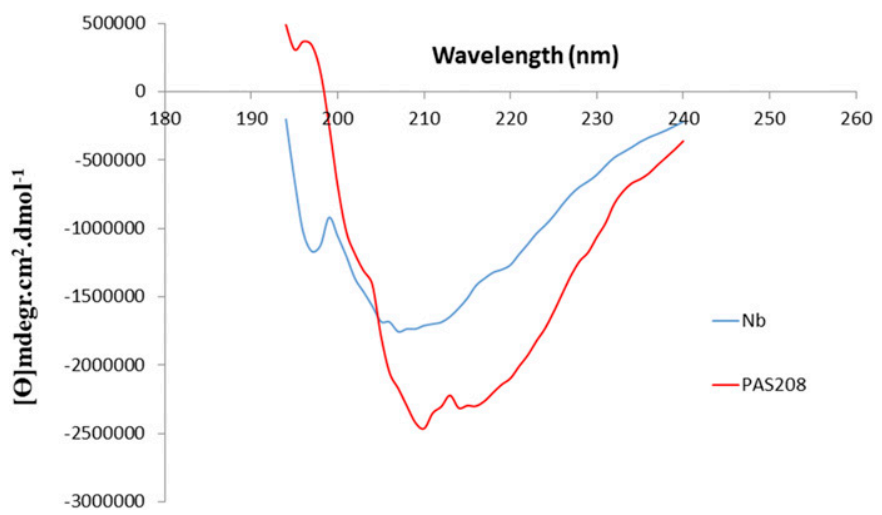

Fig. 4. Secondary structure analysis of PASylated nanobody and the native protein. 


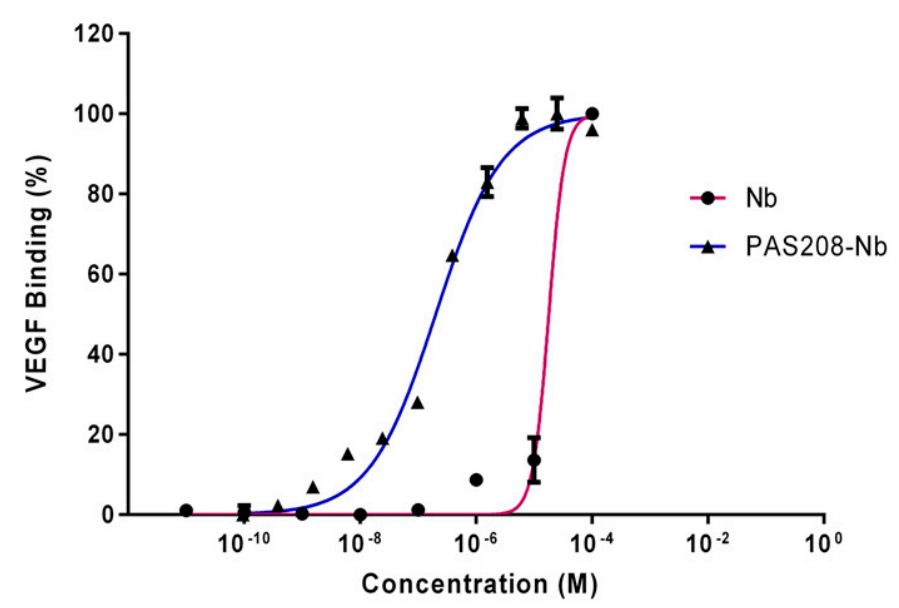

Fig. 5. Binding potency measurement. Data are presented as mean \pm S.D. from three independent measurements.

without an extra downstream process or undesired effects on the native protein. As shown in our previous study, PAS\#208 also significantly increased the $\mathrm{T}_{1 / 2}$ of the native protein by a factor of 14 after a single dose intravenous administration. Similar to PAS\#1(200)-Nb, the pharmacokinetic profile of PAS\#208-Nb showed a slower biexponential decay than the native protein. This phenomenon can be explained by random chain behavior of PAS\#208 sequence and an increase in the hydrodynamic volume of nanobody after fusion to PAS\#208
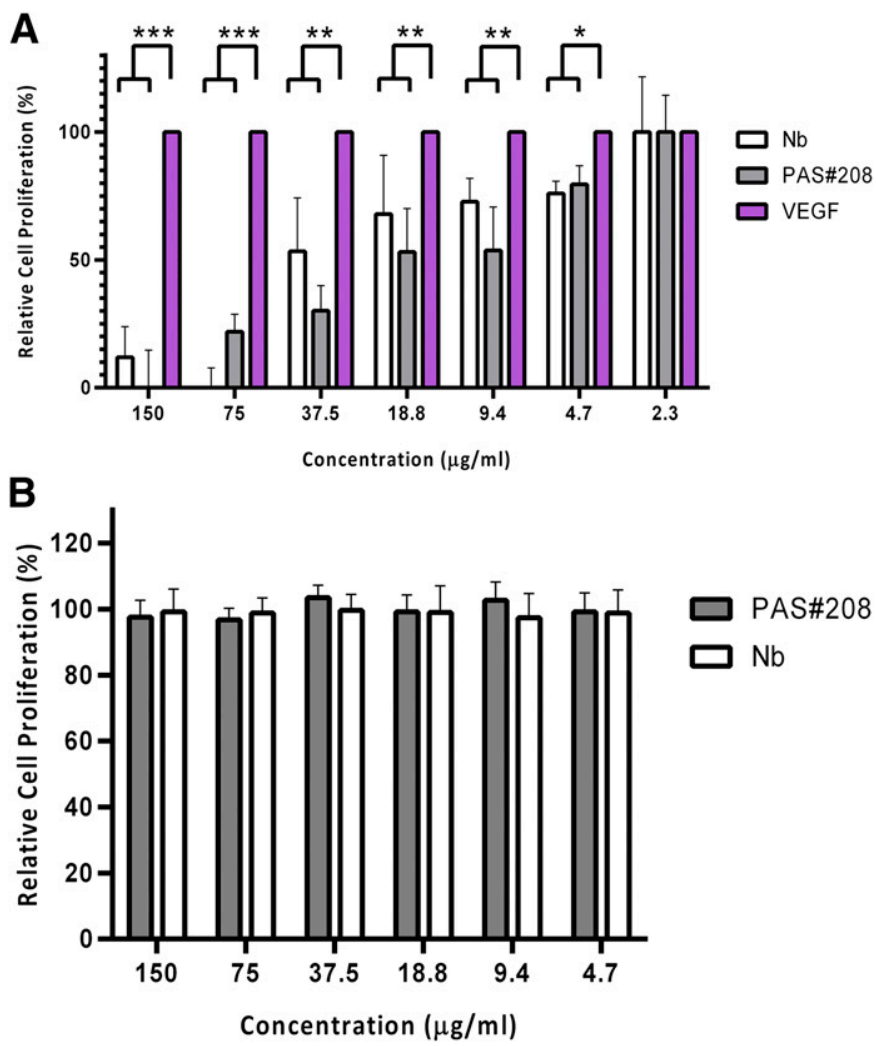

Fig. 6. VEGFA neutralization of PAS\#208-Nb and nanobody on (A) MDAMB-231 and (B) HEK293. Data are presented as mean \pm S.D. from three independent measurements. The asterisks indicate the level of significance between groups $(* * * P<0.001$; $* * P<0.01$; $* P<0.05)$.

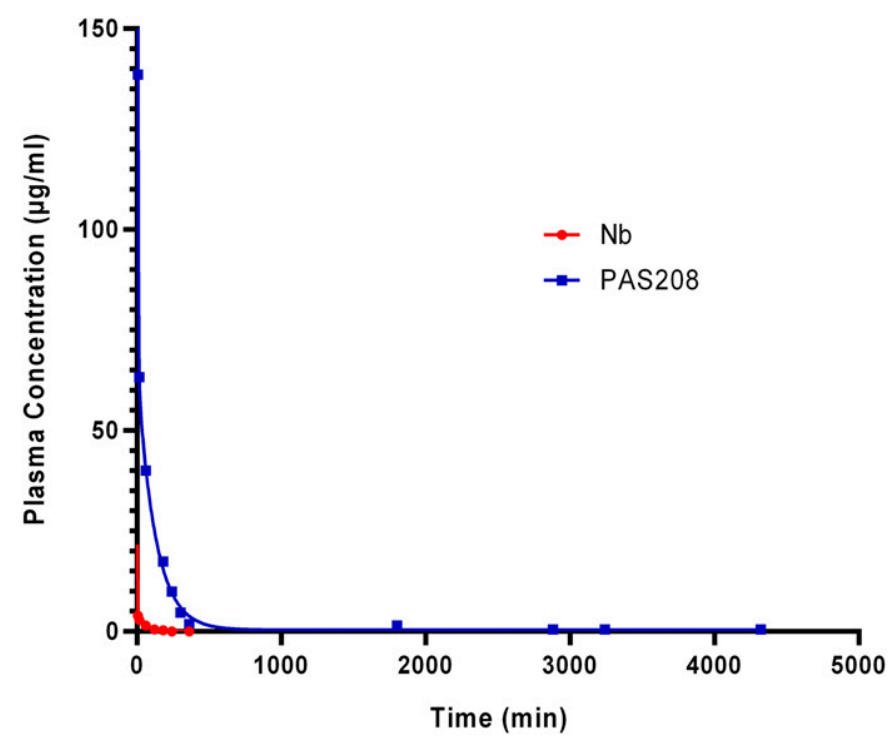

Fig. 7. Pharmacokinetic behavior of PAS\#208-Nb and the native protein after an intravenous injection of $5 \mathrm{mg} / \mathrm{kg}$ protein in mice. Nanobody was not measured in the plasma after 6 hours, whereas PAS\#208-Nb was detectable in the circulation even at 72-hour follow-up.

sequence that leads to lower glomerular filtration rate in the kidney. Such pharmacokinetic enhancements were also observed in PAS\#1(200) in our previous studies (Hedayati et al., 2017; Khodabakhsh et al., 2018). The residence of the protein in the circulation and high permeability into tumor tissues increases in-site drug concentration and enhances the efficacy of treatment.

\section{Conclusion}

In the current study, for the first time, we developed a new PAS sequence that has comparable physicochemic, biologic, and pharmacokinetic features to the previously reported PAS\#1(200) sequence. The in vitro data of our study indicated that, despite adding PAS\#208 sequence to the nanobody at the gene level, this sequence did not show any deleterious effect on the binding potency as well as neutralizing activity of the native protein. Moreover, this sequence had no cytotoxicity on the normal cells used in the study. Surprisingly, after just one dose administration of PAS\#208-Nb to the mice, the sequence remarkably improved pharmacokinetic parameters, including terminal half-life, AUC, and the volume of distribution as well as clearance due to an increase in the hydrodynamic volume of the native protein. The simplicity as well as superior effects on half-life extension make PAS\#208 sequence a novel sequence for in vivo pharmacokinetic enhancement of recombinant small proteins.

TABLE 1

Pharmacokinetic parameters of PAS\#208-Nb and the native protein calculated by the linear trapezoidal method

\begin{tabular}{lrrrrrr}
\hline Protein & $\begin{array}{c}\mathrm{T}_{1 / 2} \\
(\mathrm{~min})\end{array}$ & $\begin{array}{c}{[\mathrm{AUC}]_{0-\infty}} \\
(\mu \mathrm{g} . \mathrm{min} / \\
\mathrm{ml})\end{array}$ & $\begin{array}{c}\mathrm{V}_{\mathrm{d}} \\
(\mathrm{ml})\end{array}$ & $\begin{array}{c}\text { Clearance } \\
(\mathrm{ml} / \mathrm{min})\end{array}$ & $\begin{array}{c}\text { Elimination } \\
\text { Rate Constant } \\
(\beta)\left(\mathrm{min}^{-1}\right)\end{array}$ & $\begin{array}{c}\mathrm{PK} \\
\text { Factor }\end{array}$ \\
\hline $\begin{array}{c}\text { Nanobody } \\
\text { PAS\#208- } \\
\text { Nb }\end{array}$ & 69 & 483 & 30.4 & 0.006815 & 0.014 & 1 \\
\hline
\end{tabular}




\section{Acknowledgments}

The authors wish to thank Javid Biotechnology Institute for its technical support.

\section{Authorship Contributions}

Participated in research design: Khodabakhsh, Cohan.

Conducted experiments: Khodabakhsh, Mehdizadeh, Khosravy, Vafabakhsh, Karami, Cohan.

Performed data analysis: Khodabakhsh, Salimian, Cohan.

Wrote or contributed to the writing of the manuscript: Khodabakhsh, Salimian, Cohan.

\section{References}

Ahmadpour S and Hosseinimehr SJ (2018) PASylation as a powerful technology for improving the pharmacokinetic properties of biopharmaceuticals. Curr Drug Deliv 15:331-341.

Bradford MM (1976) A rapid and sensitive method for the quantitation of microgram quantities of protein utilizing the principle of protein-dye binding. Anal Biochem 72:248-254.

Brandl F, Merten H, Zimmermann M, Béhé M, Zangemeister-Wittke U, and Plückthun A (2019) Influence of size and charge of unstructured polypeptides on pharmacokinetics and biodistribution of targeted fusion proteins. J Control Release 307:379-392.

Cohan RA, Madadkar-Sobhani A, Khanahmad H, Roohvand F, Aghasadeghi MR, Hedayati MH, Barghi Z, Ardestani MS, Inanlou DN, and Norouzian D (2011) Design, modeling, expression, and chemoselective PEGylation of a new nanosize cysteine analog of erythropoietin. Int $J$ Nanomedicine 6:1217-1227.

Dingermann T (2013) Book review: therapeutic proteins: strategies to modulate their plasma half-lives. Biotechnol J 8:163-164.

Ecker DM, Jones SD, and Levine HL (2015) The therapeutic monoclonal antibody market, MAbs pp 9-14, Taylor \& Francis, Milton Park, Abingdon-on-Thames, Oxfordshire, United Kingdom.

Gebauer M and Skerra A (2018) Prospects of PASylation ${ }^{\circledR}$ for the design of protein and peptide therapeutics with extended half-life and enhanced action. Bioorg Med Chem 26:2882-2887.

Hedayati MH, Norouzian D, Aminian M, Teimourian S, Ahangari Cohan R, Sardari S, and Khorramizadeh MR (2017) Molecular design, expression and evaluation of PASylated human recombinant erythropoietin with enhanced functional properties. Protein $J$ 36:36-48.

Hess B, Kutzner C, van der Spoel D, and Lindahl E (2008) GROMACS 4: algorithms for highly efficient, load-balanced, and scalable molecular simulation. $J$ Chem Theory Comput 4:435-447.

Hoefman S, Ottevaere I, Baumeister J, and Sargentini-Maier ML (2015) Pre-clinical intravenous serum pharmacokinetics of albumin binding and non-half-life extended Nanobodies®. Antibodies 4:141-156.

Iezzi ME, Policastro L, Werbajh S, Podhajcer O, and Canziani GA (2018) Singledomain antibodies and the promise of modular targeting in cancer imaging and treatment. Front Immunol 9:273.

Khodabakhsh F, Norouzian D, Vaziri B, Ahangari Cohan R, Sardari S, Mahboudi F, Behdani M, Mansouri K, and Mehdizadeh A (2018) Development of a novel nanosized anti-VEGFA nanobody with enhanced physicochemical and pharmacokinetic properties. Artif Cells Nanomed Biotechnol 46:1402-1414.

Kim JY, Ahn SH, Kang ST, and Yoon BJ (2006) Electrophoretic mobility equation for protein with molecular shape and charge multipole effects. J Colloid Interface Sci 299:486-492.

Kontermann R (2012) Therapeutic Proteins: Strategies to Modulate Their Plasma Half-Lives, John Wiley \& Sons, Hoboken, New Jersey, United States.
Kontermann RE (2011) Strategies for extended serum half-life of protein therapeutics. Curr Opin Biotechnol 22:868-876.

Lin Z, Zhang Q, and Luo W (2016) Angiogenesis inhibitors as therapeutic agents in cancer: challenges and future directions. Eur J Pharmacol 793:76-81.

Longmire M, Choyke PL, and Kobayashi H (2008) Clearance properties of nano-sized particles and molecules as imaging agents: considerations and caveats. Nanomedicine (Lond) 3:703-717.

Maleki A, Madadkar-Sobhani A, Roohvand F, Najafabadi AR, Shafiee A, Khanahmad H, Cohan RA, Namvar N, and Tajerzadeh H (2012) Design, modeling, and expression of erythropoietin cysteine analogs in Pichia pastoris: improvement of mean residence times and in vivo activities through cysteine-specific PEGylation. Eur J Pharm Biopharm 80:499-507.

Mendler CT, Feuchtinger A, Heid I, Aichler M, D'Alessandria C, Pirsig S, Blechert B, Wester H-J, Braren R, Walch A, et al. (2016) Tumor uptake of anti-CD20 Fabs depends on tumor perfusion. J Nucl Med 57:1971-1977.

Morath V, Bolze F, Schlapschy M, Schneider S, Sedlmayer F, Seyfarth K, Klingenspor M, and Skerra A (2015) PASylation of murine leptin leads to extended plasma half-life and enhanced in vivo efficacy. Mol Pharm 12:1431-1442.

Muyldermans S (2013) Nanobodies: natural single-domain antibodies. Annu Rev Biochem 82:775-797.

Podust VN, Balan S, Sim B-C, Coyle MP, Ernst U, Peters RT, and Schellenberger V (2016) Extension of in vivo half-life of biologically active molecules by XTEN protein polymers. J Control Release 240:52-66.

Roy A, Kucukural A, and Zhang Y (2010) I-TASSER: a unified platform for automated protein structure and function prediction. Nat Protoc 5:725-738.

Schellenberger V, Wang CW, Geething NC, Spink BJ, Campbell A, To W, Scholle MD, Yin Y, Yao Y, Bogin O, et al. (2009) A recombinant polypeptide extends the in vivo half-life of peptides and proteins in a tunable manner. Nat Biotechnol 27: 1186-1190.

Schlapschy M, Binder U, Börger C, Theobald I, Wachinger K, Kisling S, Haller D, and Skerra A (2013) PASylation: a biological alternative to PEGylation for extending the plasma half-life of pharmaceutically active proteins. Protein Eng Des Sel 26:489-501.

Schmidt SR (2013) Fusion Protein Technologies for Biopharmaceuticals: Applications and Challenges, John Wiley \& Sons, Hoboken, New Jersey, United States.

Siegel RL, Miller KD, and Jemal A (2019) Cancer statistics, 2019. CA Cancer J Clin 69:7-34.

Skerra A, Theobald I, and Schlapschy M (2013) Biological active proteins having increased in vivo and/or in vitro stability. US Patent Number 8563521, United States Patent and Trademark Office.

Van Audenhove I and Gettemans J (2016) Nanobodies as versatile tools to understand, diagnose, visualize and treat cancer. EBioMedicine 8:40-48.

Van Roy M, Ververken C, Beirnaert E, Hoefman S, Kolkman J, Vierboom M Breedveld E, 't Hart B, Poelmans S, Bontinck L, et al. (2015) The preclinical pharmacology of the high affinity anti-IL-6R Nanobody® ALX-0061 supports its clinical development in rheumatoid arthritis. Arthritis Res Ther 17:135.

Vugmeyster Y, Entrican CA, Joyce AP, Lawrence-Henderson RF, Leary BA, Mahoney CS, Patel HK, Raso SW, Olland SH, Hegen M, et al. (2012) Pharmacokinetic, biodistribution, and biophysical profiles of TNF nanobodies conjugated to linear or branched poly(ethylene glycol). Bioconjug Chem 23:1452-1462.

Zirlik K and Duyster J (2018) Anti-angiogenics: current situation and future perspectives. Oncol Res Treat 41:166-171.

Zvonova EA, Ershov AV, Ershova OA, Sudomoina MA, Degterev MB, Poroshin GN, Eremeev AV, Karpov AP, Vishnevsky AY, and Goldenkova-Pavlova IV (2017) PASylation technology improves recombinant interferon- $\beta 1 \mathrm{~b}$ solubility, stability, and biological activity. Appl Microbiol Biotechnol 101:1975-1987.

Address correspondence to: Reza Ahangari Cohan, Department of Nanobiotechnology, New Technologies Research Group, Pasteur Institute of Iran, Tehran, Iran 1316943551. E-mail: cohan_r@pasteur.ac.ir 